\title{
Quantification of long cane usage characteristics with the constant contact technique
}

\author{
Yeongmi Kim ${ }^{1,3}$, Arturo Moncada Torres ${ }^{1}$, Jonas Furrer $^{1}$, Markus Riesch ${ }^{2}$, Roger Gassert ${ }^{1, *}$ \\ ${ }^{1}$ Rehabilitation Engineering Lab, ETH Zurich, Switzerland \\ ${ }^{2}$ Foundation Access for All, Switzerland \\ ${ }^{3}$ Department of Mechatronics, MCI, Austria
}

* Corresponding author. Department of Health Sciences and Technology, LEO B 9.1 Leonhardstrasse 278092

Zürich, Switzerland. Tel.: +41 (0)44 63232 66. E-mail address:gassertr@ethz.ch(R. Gassert) 


\title{
Quantification of long cane usage characteristics with the constant contact technique
}

\begin{abstract}
While a number of Electronic Travel Aids (ETAs) have been developed over the past decades, the conventional long cane remains the most widely utilized navigation tool for people with visual impairments. Understanding the characteristics of long cane usage is crucial for the development and acceptance of ETAs. Using optical tracking, cameras and inertial measurement units, we investigated grasp type, cane orientation and sweeping characteristics of the long cane with the constant contact technique. The mean cane tilt angle, sweeping angle, and grip rotation deviation were measured. Grasp type varied among subjects, but was maintained throughout the experiments, with thumb and index finger in contact with the cane handle in over $90 \%$ of the time. We found large inter-subject differences in sweeping range and frequency, while the sweeping frequency showed low intra-subject variability. These findings give insights into long cane usage characteristics and provide critical information for development of effective ETAs.
\end{abstract}

Keywords: long cane usage characteristics, design guidelines, visual impairment

\section{INTRODUCTION}

According to a recent World Health Organization report (WHO, 2010), about 314 million people worldwide suffer from visual impairments, mainly caused by uncorrected refractive errors such as myopia, astigmatism, cataract or glaucoma. In the 1940s, the modern long cane was introduced to assist people with visual impairments in traveling safely and independently. Several navigation techniques, e.g. constant contact, two-point touch, diagonal and shoreline were proposed for the long cane (De Bruin, 1981; Hill and Ponder, 1976). Among these techniques, the constant contact technique - sweeping the cane side to side with the tip in contact with the ground (De Bruin, 1981), and the two-point touch technique - touching the ground with the cane tip on the opposite side of the forefoot (Hill and Ponder, 1976) are most commonly employed (LaGrow and Weessies, 1994). 
In addition to the conventional long cane, guide dogs can support safe travel of blind people with visual impairments. However, a guide dog requires a long training period to reliably perform its tasks, generates important costs, and requires care and attention from the blind or visually impaired person. Both methods do not entirely guarantee safe travel as the sensing range remains below that of sighted people, and the information bandwidth is rather limited. In addition, head-level obstacles or distant drop-offs are not easily detected. With the evolution of sensing and display technologies, Electronic Travel Aids (ETAs) were introduced to extend the sensing range beyond that of a conventional long cane and to present enriching information about the environment through auditory or haptic channels. ETAs can be broadly divided into two categories, i.e. navigation aids and mobility aids. Navigation aids provide information for global orientation to find the way from the current location to a desired destination (Chandler and Worsfold, 2013) by means of Global Positioning System (GPS), laser range finders or Radio-Frequency Identification (RFID) tags (BrailleNote GPS; Kulyukin et al., 2004; Sánchez, 2007). Mobility aids in return present vicinity information to the user. Some mobility aids indicate the clear path (Ulrich and Borenstein, 2001; Shoval et al., 1998) while others provide location information on static and moving obstacles to prevent collisions (Goldstein and Wiener, 1981; La Grow, 1999; Benjamin, 1971; Hoyle and Waters, 2008; Lee et al., 2014; MiniGuide). While a few hand held (MiniGuide; Bissitt and Heyes, 1980) and wearable mobility aids (iGlasses; Shoval et al., 1998; Meers and Ward, 2005) have been developed over the past decades, the majority of ETAs were designed to be mounted on a long cane (Kim et al., 2015; Wang and Kuchenbecker, 2012; Gallo et al., 2010; Kim et al., 2009b; Hoyle and Waters, 2008; Farcy and Bellik, 2002). Wearable ETAs require additional donning which often become bulky. In the case of hand held ETAs, it is possible to fail to detect dangerous obstacles and receive false alarm feedback when holding ETA with incorrect orientation. To avoid this issue, an auditory alarm was employed to assist users to maintain correct orientation of the hand held ETA (Bissitt and Heyes, 1980). ETAs mounted on a long cane benefits to utilize a conventional cane which is familiar to long cane users, delivers ground surface information and detects obstacle within long cane preview range.

ETAs not only provide additional alarms for obstacles that are not detected by a long cane, but also improve ambulation. Clark-Carter et al. (1986) measured walking speed with the Sonic Pathfinder (Heyes, 1984) with several preview distances (detection range of the ETA). The results showed that the walking speed was significantly increased when obstacle information was provided from up to $3.5 \mathrm{~m}$ away in comparison to the conventional long cane which typically reaches about $1.2 \mathrm{~m}$ from the body. ETAs may therefore enhance the perceived level of safety, confidence and efficiency of navigation of blind and visually impaired users by increasing the speed at which they can walk with confidence. Lee et al. (2014) compared task completion time and number of collisions with obstacles placed along an experimental track in 3 conditions (i.e. using a long cane, using a body-mounted ETA as well as a 
combination of long cane and ETA). They found that combining the long cane with the ETA resulted in a reduction of both the task completion time and the number of collisions with obstacles.

Since the first device was commercialized in the 1960s (Kay, 1964: K-Sonar), numerous ETAs have been proposed, developed and marketed. Despite their advantages, visually impaired persons still rely largely on the traditional long cane, most likely due to the drawbacks of existing ETAs, resulting from an unergonomic interface, overload of sensory channels, non-intuitive or unreliable feedback, excessive weight, long training periods and high cost. Several of these drawbacks can be explained by a technologydriven approach that has been taken in many previous projects, compared to a more user-driven approach, which should be followed in our opinion, and for which this study further paves the way.

Deriving meaningful design requirements is a significant step towards the development of efficient ETAs that can complement the conventional long cane and be widely employed. Therefore, understanding navigation strategies and other characteristics of long cane usage can inform the design of efficient ETAs. Previous studies investigated biomechanical parameters related to the navigation of people with visual impairments. Nakamura (1997) analyzed the gait of congenitally blind, late blind and sighted people. The results showed that the congenitally blind have the slowest walking speed, shortest stride length, and longest stance phase compared to late blind and sighted people. Ramsey et al. (1999) explored kinematic parameters (i.e. gait velocity, stride length, and hip flexion velocity), showing that these differ in attention-demanding environments, when approaching drop-offs, or during normal walking. Johnson et al. (1998) found longer mediolateral displacement, and higher mean cane velocity in a visually impaired group compared to a mobility instructor group. To increase the efficiency and safety of white cane usage in visually impaired persons, some research focused on optimizing the design of the conventional long cane or the improving navigation techniques. Kim et al. (2009a) investigated the performance of detecting drop-offs between the two widely used cane techniques. The results of the experiment showed that the constant contact technique provides significantly higher drop-off detection rate than the two-point touch technique. To examine the usefulness of tapping sounds generated by a long cane for object detection using semi-active echolocation, different cane types and object sizes were tested (Schenkman and Jansson, 1986). While the size of objects plays an important role in echolocation, the cane type did not affect the performance. These findings and measurements of previous studies can be useful to derive guidelines for orientation and mobility $(\mathrm{O} \& \mathrm{M})$ instructors to select an appropriate cane technique and cane type based on the needs of visually impaired persons. These are also valuable for engineers wishing to develop effective ETAs.

Further studies which are more relevant to design an ETA have been carried out. Wall and Ashmead (2002) investigated variable deviation of the two-point touch technique between classical guideline and actual usages. Hand position was located at the right side of the middle line for right handed participants. 
This implies that angle of sensors on a long cane can be adjusted to the right side to detect forward obstacles. Cane arc width which is related to cane yaw angle is greater than a recommended width. Wrist angle has a great influence on the roll angle. When a cane rolls, it might detect ground as an obstacle. It is also necessary to measure a range of roll angle because not only wrist roll angle but also wrist bending angle and cane rotation in side of hand can change cane roll angle. An analogous experiment was carried out by using the two-point touch technique (Bongers et al., 2002). Among several variables, cane tip height has impact on drop-off detection in ETAs (Pyun et al., 2013; Benjamin, 1971). Since a cane was lifted higher than recommended height while using the two-point touch technique, additional sensors to detect cane height to avoid false alarm drop-off signal could be considered for designing ETAs. The angle between cane and the ground helps to determine angle of sensors mounted on a long cane. However, continuous observation of this angle would be also useful for determining and placement of sensors in ETAs. Furthermore, cane orientations or variation of cane height which influence sensing ranges of sensors can be varied depending on long cane techniques

There are additional significant variables of cane usage to provide design guidelines for ETAs as follows.

- Where do fingers contact on the cane handle and do they vary during ambulation?

- What are the mean three cane orientation angles? Do these angles vary while walking with a cane and between subjects?

- Are sweeping characteristics different between long cane users?

To the best of our knowledge, these parameters critical for the design and placement of sensor and display technologies for ETAs, such as cane orientation, sweeping range, grasp types and finger contact locations have so far been unexplored. Additionally, different long cane techniques result in different variables of cane usage.

Therefore, we investigate characteristics of long cane usage with the constant contact cane technique to gain a better understanding of grasping, sweeping and cane orientation variables allowing to derive guidelines for the design of efficient ETAs. Based on these measurements, design guidelines for the development of efficient ETAs are formulated.

\section{MATERIALS AND METHODS}

\subsection{Subjects}


Ten visually impaired subjects were recruited through the foundation Access for All, a Swiss nonprofit organization. Subjects voluntarily participated in the experiments and signed a written consent form after receiving a full explanation of the experimental procedure. The study was previously approved by the local research ethics committee. Nine subjects (subjects 1 to 9) took part in experiment I, while seven subjects (subjects 4 to 10) participated in experiment II. Table 1 summarizes the subject demographics. Participants were aged from 22 to 65 (mean age: 37.3, Standard Deviation (SD): 15.9), all right handed and held their cane in the right hand. All participants were legally blind with a visual acuity below 20/200 (Koestler, 2004) and were experienced long cane users. All subjects used a folding cane consisting of several segments. The primary cane technique indicates the preferred method when navigating with a long cane, but all subjects responded that they adapted the cane technique depending on the ground condition or other environmental circumstances. Eight subjects reported that they mainly employ the constant contact technique. The other two subjects primarily employ the two-point touch technique, but are also familiar with the constant contact technique.

\section{Table 1}

Characteristics of participants

\begin{tabular}{lcccccl}
\hline $\begin{array}{l}\text { Subject } \\
\text { No. }\end{array}$ & Gender & $\begin{array}{c}\text { Age } \\
{[\text { years }]}\end{array}$ & $\begin{array}{c}\text { Height } \\
{[\mathrm{m}]}\end{array}$ & Onset of blindness & $\begin{array}{c}\text { Primary long cane } \\
\text { technique }\end{array}$ & Residual vision \\
\hline 1 & Male $^{1}$ & 31 & 1.54 & Congenitally blind & Two-point touch & No light perception \\
2 & Female $^{1}$ & 22 & 1.79 & Congenitally blind & Constant contact & Perception of color \\
3 & Female $^{1}$ & 25 & 1.62 & Congenitally blind & Constant contact & No light perception \\
4 & Male $^{1,2}$ & 56 & 1.79 & Congenitally blind & Constant contact & Perception of light \\
5 & Male $^{1,2}$ & 65 & 1.69 & Congenitally blind & Constant contact & No light perception \\
6 & Male $^{1,2}$ & 25 & 1.88 & Congenitally blind & Constant contact & Perception of light \\
7 & Male $^{1,2}$ & 23 & 1.86 & Congenitally blind & Constant contact & Perception of color \\
8 & Male $^{1,2}$ & 54 & 1.82 & Congenitally blind & Constant contact & No light perception \\
9 & Male $^{1,2}$ & 29 & 1.85 & Congenitally blind & Two-point touch & No light perception \\
10 & Male $^{2}$ & 43 & 1.82 & Late blindness & Constant contact & Perception of light \\
\hline${ }^{1}$ These subjects participated in experiment I. ${ }^{2}$ These subjects participated in experiment II. &
\end{tabular}

\subsection{Experiment I - Cane grasping and sweeping characteristics}




\subsubsection{Procedure}

Experiment I mainly focused on quantifying grasping and sweeping characteristics of the constant contact technique. In a first step, static measurements were carried out in an empty room. At the beginning of the experiment, the length of the long cane of each participant was recorded. They were then asked to naturally sweep their own long cane as usually done during navigation, but while walking in place. After five cycles of sweeping, subjects were asked to pause the movement when the cane was pointed forward. The distance between the cane tip and the heel was measured with a tape measure. In a second step, the placement of the fingers on the cane handle and the location of the flat surface of the cane handle were documented as illustrated in Fig. 1. Note that often the cane handle has one flat side where the index finger is placed during conventional O \& M training (Cutter, 2007).

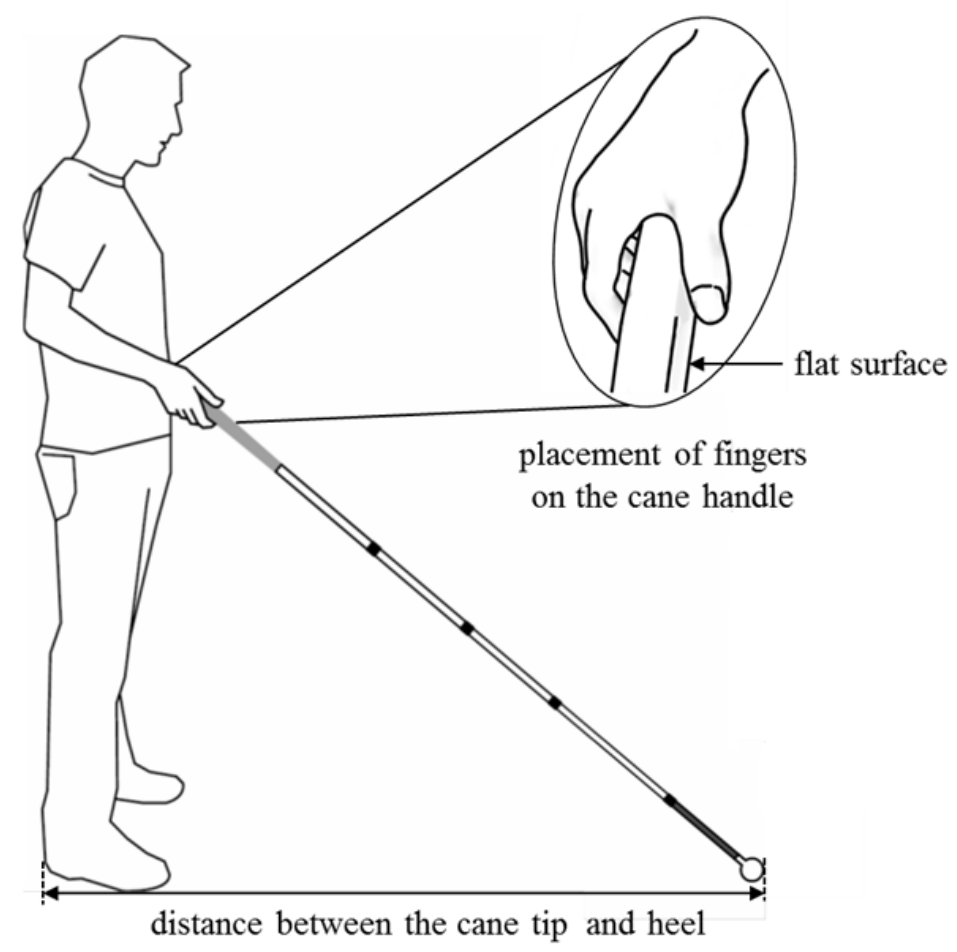

Fig. 1. Static measurements to investigate cane length, and grasp type.

After the static measurements, dynamic measurements were carried out with an instrumented long cane. Before starting the measurements, a small portable video camera $\left(\mathrm{C}_{1}\right)\left(\right.$ TrendMicro, BRAUN $\left.{ }^{\circledR}\right)$ and a ReSense (RS) inertial measurement unit (IMU) module, a low power, small-sized and light-weight inertial measurement unit consisting of a 3-axis accelerometer and a 3-axis gyroscope (Leuenberger and Gassert, 2011), were tightly attached to the cane of each subject as depicted in Fig. 2. 
The camera was positioned at a distance $\left(\mathrm{d}_{\mathrm{Cl}}\right)$ of $40 \mathrm{~cm}$ from the upper edge of the cane handle at an angle $\left(\theta_{\mathrm{C} 1}\right)$ of $110^{\circ}$, pointing toward the subject in order to film the subject's grip on the cane handle. The IMU module was attached to the cane $80 \mathrm{~cm}$ from the tip $\left(\mathrm{d}_{\mathrm{RS}}\right)$, and aligned with $\mathrm{C}_{1}$ in order to measure the sweeping velocity and frequency. An additional camera $\left(\mathrm{C}_{2}\right)$ was installed at a few meters distance to monitor the experimental scene.

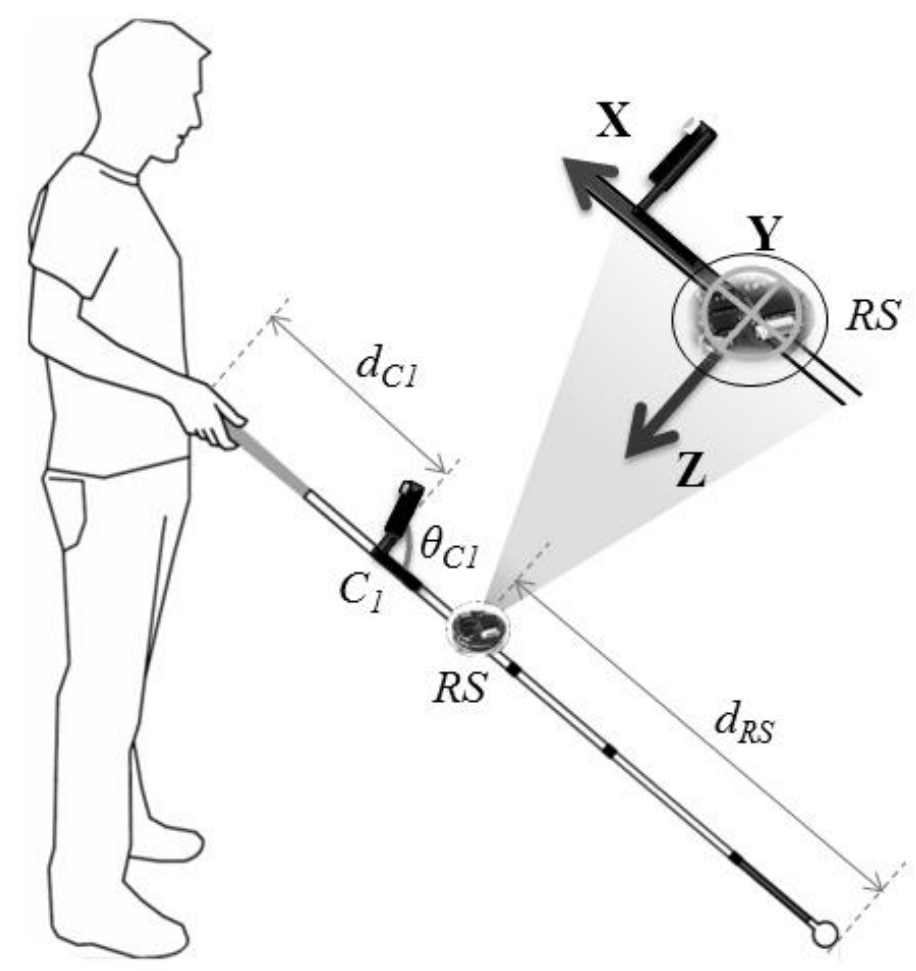

Fig. 2. Long cane setup for the dynamic measurements of experiment I, showing the placement and alignment of the 6DOF IMU (RS) as well as the hand-facing camera $\left(\mathrm{C}_{1}\right)$.

The experiment took place along an obstacle free straight path. Two lines were marked on the ground with an interval of $7 \mathrm{~m}$ of distance, $\mathrm{d}_{\text {walk }}$, to examine the walking speed. Each subject was instructed to stand at the start position and lift the long cane three times to indicate the start of the events and to synchronize the sensor data with the recordings of the external camera $\mathrm{C}_{2}$. The subject was then asked to walk straight using the constant contact technique until the experimenter gave a verbal instruction to stop. This procedure was repeated twice, with the video cameras and the IMU modules capturing data throughout the entire trials without pause. Although the experimental setup was obstacle free, subjects were not informed about obstacles to ensure that they would walk normally with their own long cane. 


\subsubsection{Data analysis}

Walking speed $s_{\text {walk }}$ was calculated from the video captured by $C_{2}$ by multiplying $d_{\text {walk }}$ by the camera frame rate $\mathrm{fr}_{\text {rate }}$ and dividing by the difference of the last frame $\mathrm{Nfr}_{\text {end }}$ and the first frame $\mathrm{Nfr}_{\text {begin }}$, according to Equation 1.

$$
s_{\text {walk }}=\frac{d_{\text {walk }} f r_{\text {rate }}}{N f r_{\text {end }}-N f r_{\text {begin }}}
$$

In addition to the walking speed, sweeping frequency and mean sweeping angular velocity were derived from the data stored on the IMU module. The gyroscope z-axis signal gyro $_{z}$ was digitally processed and analyzed, as this axis yields information regarding the sweeping movement of the cane. First, gyro $_{z}$ was trimmed based on the peak signals and captured videos to synchronize gyroscope data with the video epochs. Second, the mean of the signal was subtracted from the raw signal in order to remove any undesired drift. Then, the signal was filtered $\left(\mathrm{gyro}_{\mathrm{zFilt}}\right)$ with a $4^{\text {th }}$ order IIR Butterworth digital low-pass filter to remove any high frequency components. A cut-off frequency $\mathrm{f}_{\mathrm{c}}$ of $5 \mathrm{~Hz}$ was chosen empirically to remove noise. We defined a sweep cycle as the complete movement of the cane from the initial position at the right-most point and back from the left-most point to the initial position of the subject's sweeping range. Therefore, sweeping frequency $\mathrm{f}_{\text {sweep }}$ indicates the number of complete sweep cycles performed per second. Since in both the left and right-most points of the sweeping range the angular velocity of the cane movement is zero, $\mathrm{f}_{\text {sweep }}$ was calculated as the quotient between the number of zero-crossings $\mathrm{N}_{\mathrm{zc}}$ in gyro $_{z F i l t}$ and the product of two times the signal duration $t$ in seconds, as expressed in Equation 2.

$$
f_{\text {sweep }}=\frac{N_{z c}}{2 t}
$$

Sweeping speed $\mathrm{s}_{\text {sweep }}$ was defined as the angular velocity during the sweeping period. The mean sweeping speed $\overline{\mathbf{s}}_{\text {sweep }}$ was calculated by rectifying gyro $_{z \mathrm{Filt}}$ and obtaining its mean value as shown in Equation 3.

$$
\bar{S}_{\text {sweep }}=\frac{\sum_{i=0}^{N-1} \mid \text { gyro }_{z \text { Filt }}(i) \mid}{N}
$$

The camera $\mathrm{C}_{1}$ served the purpose of investigating how cane users hold their canes and to identify the proportion of contact vs. non-contact of fingers on the cane handle during navigation. Also, grasp changes over time could be observed. This is relevant for the placement of interface buttons or a haptic display 
that could convey information about the environment via vibration or other haptic feedback from the handle to the user.

Analogous to the static measurements, positions of the index finger and thumb were examined during walking with the instrumented cane. We calculated the distributions of placement of the thumb and index finger at three locations (i.e. side, top, and bottom) through visual observation by $\mathrm{C}_{1}$ with frame rate of 30 frames/second, while the fingers contacted the cane handle. Contact free phases of the index finger and thumb were also examined during walking. These are presented when the fingertip and distal interphalangeal (DIP) joint of the index finger are not both in contact with the cane. For the thumb, the contact free condition was defined when both the fingertip and interphalangeal joint (IP) were removed from the handle. When the fingertip was in contact with the handle, but the DIP and IP joints were not, this was also defined as finger contact.

\subsection{Experiment II - Cane orientation angles}

In experiment I, grasping and sweeping characteristics were investigated with the help of an IMU and cameras. To quantify cane orientation, a further experiment was conducted using an optoelectronic motion tracking system (QTM/Oqus, Qualisys AB) to obtain accurate cane orientation angles.

\subsubsection{Apparatus}

Ten Oqus 3 cameras were mounted on a frame surrounding the experimental platform $(6.6 \mathrm{~m} \times 5.0 \mathrm{~m} \times$ $3.5 \mathrm{~m})$ and adjusted to cover a wide walking path and to ensure continuous tracking of all markers. In addition, four video cameras were mounted to record front, back, side and top views as a reference. The same five-section folding cane of $1.3 \mathrm{~m}$ length was used for all subjects in this experiment. A threedimensional arrangement of 6 passive reflective markers was attached to the long cane $(80 \mathrm{~cm}$ from the cane tip). Non-reflective tape was employed to avoid reflection artifacts resulting from tracking system illumination striking the cane surface, and to prevent rotation of the foldable segments of the cane against each other.

\subsubsection{Procedure}

Experimenter monitoring, familiarization sessions, and verbal instructions during each session ensured the safety of participants during the experiments. Each subject was instructed to employ the instrumented 
long cane along a straight path from the marked starting position to the marked destination. Subjects were instructed to use the constant contact technique while walking, as this cane technique is the majority of the primary long cane technique and could be performed by all participants. Participants received a verbal start signal from the experimenter and walked along a straight path until they received a verbal cue to stop walking. Subjects completed two familiarization trials of the straight walk with the instrumented cane before the three experiment trials and data recording.

\subsubsection{Data analysis}

To calculate the position and orientation of the long cane, the origin of the right-handed Cartesian global coordinate system was chosen at the fixed starting location, with the $\mathrm{X}$ and $\mathrm{Y}$ axes along the ground plane and the $\mathrm{Z}$ axis pointing upwards. A right-handed Cartesian local coordinate system with origin on the cane, $80 \mathrm{~cm}$ from the tip with the $\mathrm{x}$-axis along the cane, and the $\mathrm{y}$-axis pointing approximately to the left side of the subject, was further selected. Fig. 3 (a) illustrates the global and the local cane coordinate systems.

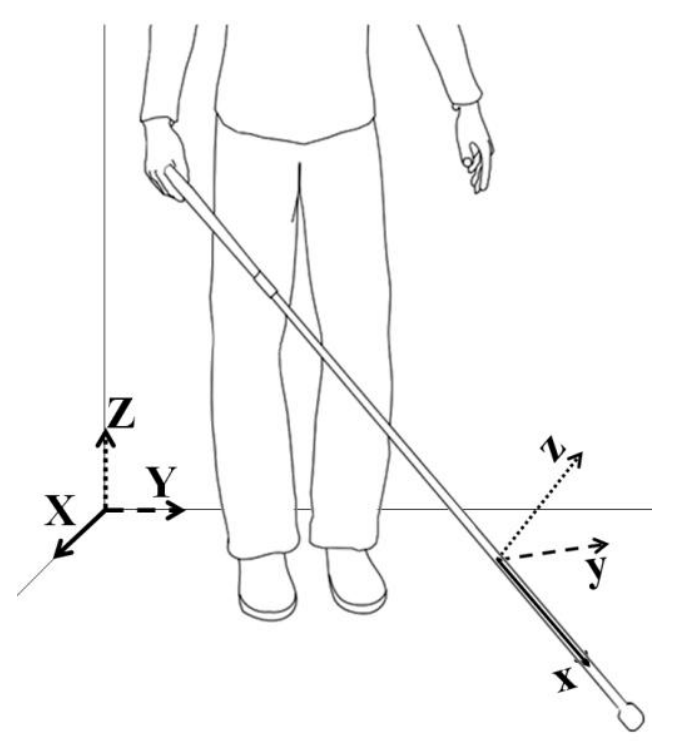

(a)
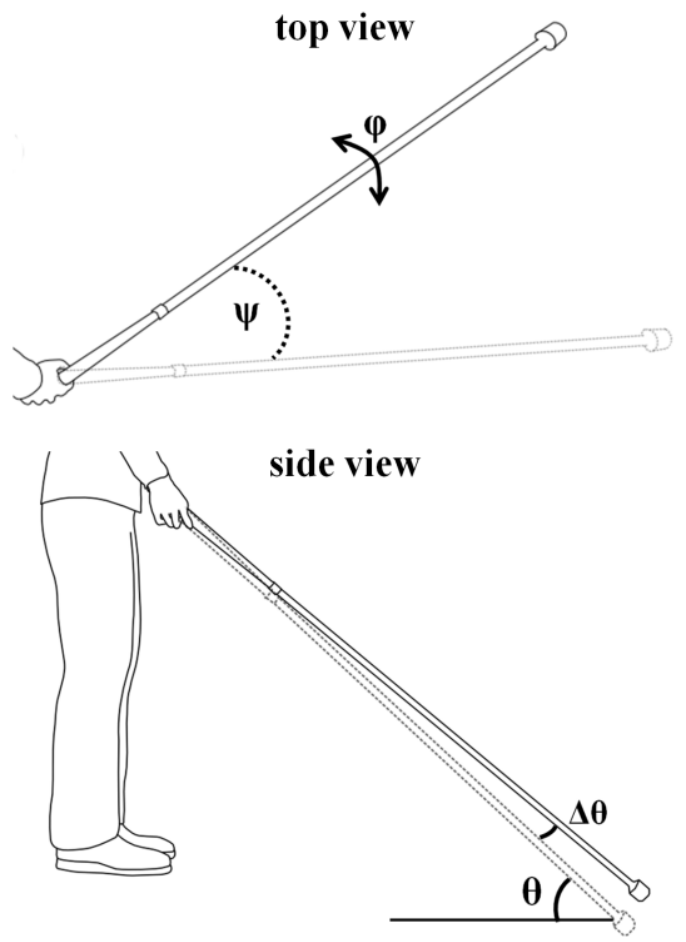

(b) 
Fig. 3. (a) Global coordinate system (X-Y-Z) and local coordinate system (x-y-z) on the cane (b) sweeping angle $(\psi)$ and cane grip rotation $(\varphi)$ (top), tilt angle $(\theta)$ and tilt angle range $(\Delta \theta)$ (bottom).

Using the optoelectric motion tracking system, the position and orientation of each individual marker and marker set were recorded at a sampling rate of $100 \mathrm{~Hz}$. A sample consisted of the position of the origin of the cane-fixed coordinates expressed in global coordinates, as well as the rotation matrix which mapped the world to local cane coordinates. The analysis of the data was performed in MATLAB (The MathWorks). From the rotation matrix, the Tait-Bryan rotation angles (intrinsic or mobile frame rotations of $\psi$ around $\mathrm{z}, \theta$ around $\mathrm{y}$ and $\varphi$ around $\mathrm{x}$ ) were calculated (Black, 2009). Based on the selected coordinate systems, we mapped the rotation angles to construct the cane movement parameters (roll: $\varphi$, pitch: $\theta$ and yaw: $\psi$ corresponding to cane grip rotation, tilt angle, and sweeping angles respectively). The sweeping angle indicates how far the cane is rotated around the z-axis in a single sweep from one side to the other. It was calculated as the absolute difference between two consecutive local extremes in the yaw angle $\psi$ over time, illustrated in Fig. 3 (b). As shown in Fig. 3 (b), the pitch angle $\theta$ was defined as the angle between the cane axis and the ground. The tilt angle range was calculated as the difference between the maximum and minimum pitch angle within one trial. Similar to the tilt angle range, the cane grip rotation range was also computed as the difference between the maximum and minimum roll angle $\varphi$, as depicted in Fig. 3 (b).

\section{Results}

\subsection{Static measurement of long cane usage and grasping characteristics}

Table 2 summarizes the results of the static measurements of long cane usage. The cane length ranged from $1.15 \mathrm{~m}$ to $1.5 \mathrm{~m}$ (mean: 1.38 , SD: 0.11 ) and the mean distance between cane tip and heel was $1.2 \mathrm{~m}$. To identify the position of the thumb and index finger, we defined three possible areas on the cane handle where these two fingers can be placed. As all subjects were right handed, side refers to the left side of the cane for the thumb, and to the right side of the cane for the index finger. Eight subjects placed their thumb on the side of the cane, while one subject placed the thumb on the top of the handle. In comparison, the placement of the index finger was more variable. Six subjects stabilized the cane from the side with the index finger, while one subject placed the index finger on top of the cane. In the case of rest two subjects, the tip of index finer was placed on the bottom of the cane handle by wrapping the hand around the handle with the index finger. Additionally, the finger that contacts the flat surface of the cane handle was 
identified. More than half of the subjects placed the index finger on the flat side, while three subjects placed the thumb there. Note that the cane handle of subject 4 did not have a flat surface.

\section{Table 2}

Static measurement of long cane usage

\begin{tabular}{cccccc}
\hline $\begin{array}{c}\text { Subject } \\
\text { No. }\end{array}$ & $\begin{array}{c}\text { Static } \\
\text { thumb } \\
\text { position }\end{array}$ & $\begin{array}{c}\text { Static index } \\
\text { finger position }\end{array}$ & $\begin{array}{c}\text { Finger placed } \\
\text { on flat surface }\end{array}$ & $\begin{array}{c}\text { Cane } \\
\text { length }[\mathrm{m}]\end{array}$ & $\begin{array}{c}\text { Distance between } \\
\text { the cane tip and } \\
\text { heel [m] }\end{array}$ \\
\hline 1 & Side & Side & Thumb & 1.15 & 1.05 \\
2 & Side & Bottom & Thumb & 1.45 & 1.31 \\
3 & Side & Side & Index finger & 1.31 & 1.17 \\
4 & Side & Bottom & No flat surface & 1.40 & 0.90 \\
5 & Side & Top & Index finger & 1.28 & 1.12 \\
6 & Side & Side & Thumb & 1.5 & 1.41 \\
7 & Top & Side & Index finger & 1.44 & 1.26 \\
8 & Side & Side & Index finger & 1.43 & 1.36 \\
9 & Side & Side & Index finger & 1.44 & 1.24 \\
\hline Mean & & & & 1.38 & 1.2 \\
SD & & & & 0.11 & 0.16 \\
\hline
\end{tabular}

Table 3 summarizes the grasping characteristics of the constant contact cane technique while walking with a long cane. The first two columns report the initial thumb and index finger positions on the cane handle before initiating walking. All subjects initially placed the thumb and index finger as in the static measurements of experiment I (Table 1). In the majority of cases, both the tip of the thumb (mean: 84.5\%) and the index finger (mean: 67.08\%) were placed on the side of the cane handle. Even though subjects had different preferred grasp types, these two fingers were mostly kept at the same location while walking. The mean percentage of non-contact total time was $9.24 \%$ and $3.17 \%$ for thumb and index finger, respectively. Subject 2 showed a higher percentage of non-contact state for the thumb than other subjects as the other four fingers were always curled around the cane handle, resulting in a firm grasp. During the position transition of the fingers, a non-contact situation occurred in subjects 3 and 5 . As subject 6 grasped the cane handle more loosely than others, the index finger was often not in contact with the cane handle. Due to occlusion issues when capturing the scenes from the hand-facing camera attached to the cane, the positions of the middle finger, ring finger and little finger could not be objectively assessed over the trials, but the fingers were wrapped around the handle most of time. 
Table 3

Cane grasping characteristics of constant contact cane technique during dynamic measurements

\begin{tabular}{|c|c|c|c|c|c|c|c|c|c|c|}
\hline \multirow[t]{2}{*}{$\begin{array}{l}\text { Subject } \\
\text { No. }\end{array}$} & \multirow{2}{*}{$\begin{array}{l}\text { Initial } \\
\text { position } \\
\text { of } \\
\text { thumb }\end{array}$} & \multirow{2}{*}{$\begin{array}{l}\text { Initial } \\
\text { position } \\
\text { of index } \\
\text { finger }\end{array}$} & \multicolumn{3}{|c|}{$\begin{array}{c}\text { Distribution of thumb } \\
\text { position during walking } \\
{[\%]}\end{array}$} & \multicolumn{3}{|c|}{$\begin{array}{c}\text { Distribution of index } \\
\text { finger position during } \\
\text { walking } \\
{[\%]}\end{array}$} & \multicolumn{2}{|c|}{$\begin{array}{c}\text { Proportion of } \\
\text { non-contact [\%] }\end{array}$} \\
\hline & & & Side & Top & Bottom & Side & Top & Bottom & Thumb & $\begin{array}{l}\text { Index } \\
\text { finger }\end{array}$ \\
\hline 1 & Side & Side & 100 & 0 & 0 & 68 & 0 & 32 & 1.91 & 2.03 \\
\hline 2 & Side & Bottom & 100 & 0 & 0 & 0 & 0 & 100 & 60.28 & 0 \\
\hline 3 & Side & Side & 60.55 & 29.59 & 9.86 & 99.02 & 0 & 0.98 & 15.84 & 3.96 \\
\hline 4 & Side & Bottom & 100 & 0 & 0 & 0 & 0 & 100 & 1.89 & 0 \\
\hline 5 & Side & Top & 100 & 0 & 0 & 36.69 & 63.31 & 0 & 0.57 & 2.29 \\
\hline 6 & Side & Side & 100 & 0 & 0 & 100 & 0 & 0 & 0 & 16.3 \\
\hline 7 & Top & Side & 0 & 100 & 0 & 100 & 0 & 0 & 0 & 0 \\
\hline 8 & Side & Side & 100 & 0 & 0 & 100 & 0 & 0 & 0 & 0 \\
\hline 9 & Side & Side & 100 & 0 & 0 & 100 & 0 & 0 & 2.65 & 3.97 \\
\hline$\overline{\text { Mean }}$ & & & 84.5 & 14.4 & 1.10 & 67.08 & 7.03 & 25.89 & 9.24 & 3.17 \\
\hline SD & & & 34.27 & 33.56 & 3.29 & 43.70 & 21.10 & 43.29 & 19.79 & 5.20 \\
\hline
\end{tabular}

\subsection{Sweeping characteristics}

In experiment I, the group mean of the walking speed was 1.05 (SD: 0.21) [m/s] and the mean sweeping angular velocity was 54.1 (SD: 17.26) [\% $\%$. The mean sweeping frequency was 0.78 (SD: 0.17 ) [sweeps/s], with high inter-subject variability. The minimum sweeping frequency [0.52 sweeps/s] was about $54 \%$ of the maximum frequency [0.96 sweeps/s].

\subsection{Rotation angles of the long cane}


Table 4 shows the results of the rotation angle measurements. The minimum sweeping angle refers to the group mean of the smallest sweeping angles of all subjects. The latter was determined as the mean of the smallest sweeping angles of the three trials of each subject. Although the mean minimum sweeping angle was $37.8^{\circ}$, the standard deviation was large due to the wide range of different cane usage characteristics of subjects. Furthermore, the mean sweeping angle also showed large variability among long cane users. The group mean sweeping angle was $48.63^{\circ}$, but the mean sweeping angles of individual subjects ranged from $19.63^{\circ}$ to $88.87^{\circ}$. The mean tilt angle range was $5.27^{\circ}$ and the mean tilt angle was $43.17^{\circ}$. The tilt angle showed less variation among subjects than the sweeping angle. Lastly, the mean cane grip rotation range was $26.34^{\circ}$ (SD: 8.70).

\section{Table 4}

Rotation angles of the long cane

\begin{tabular}{lccccc}
\hline & & Mean & SD & Min & Max \\
\hline Sweeping angle $(\psi)$ & Minimum angle $\left[^{\circ}\right]$ & 37.80 & 21.06 & 13.20 & 67.73 \\
& Maximum angle $\left[^{\circ}\right]$ & 62.01 & 31.45 & 27.73 & 155.17 \\
& Mean sweep angle $\left[^{\circ}\right]$ & 48.63 & 26.15 & 19.63 & 88.87 \\
Tilt angle $(\theta)$ & Range $\left[{ }^{\circ}\right]$ & 5.27 & 2.50 & 3.13 & 9.77 \\
& Minimum angle $\left[^{\circ}\right]$ & 40.28 & 6.47 & 31.03 & 48.63 \\
& Maximum angle $\left[{ }^{\circ}\right]$ & 45.54 & 4.55 & 40.1 & 51.93 \\
Cane grip rotation angle $(\varphi)$ & Mean angle $\left[{ }^{\circ}\right]$ & 43.17 & 5.17 & 37.07 & 50 \\
& Range $\left[{ }^{\circ}\right]$ & 26.34 & 8.70 & 17.27 & 39.03 \\
\hline
\end{tabular}

Fig. 4 shows plots of the measured rotation angles and mean sweeping velocity during one trial of subject 4 in Experiment II. The top graph shows the periodic angle changes over time. The circles mark the maximum and minimum angles. The second graph indicates the sweeping velocity. Similar to the sweeping angle, it is also periodic but tends to be maximal at the half-time between the minimum and maximum sweeping angles. The tilt and roll angle also show periodic patterns, as depicted in the last two graphs. 


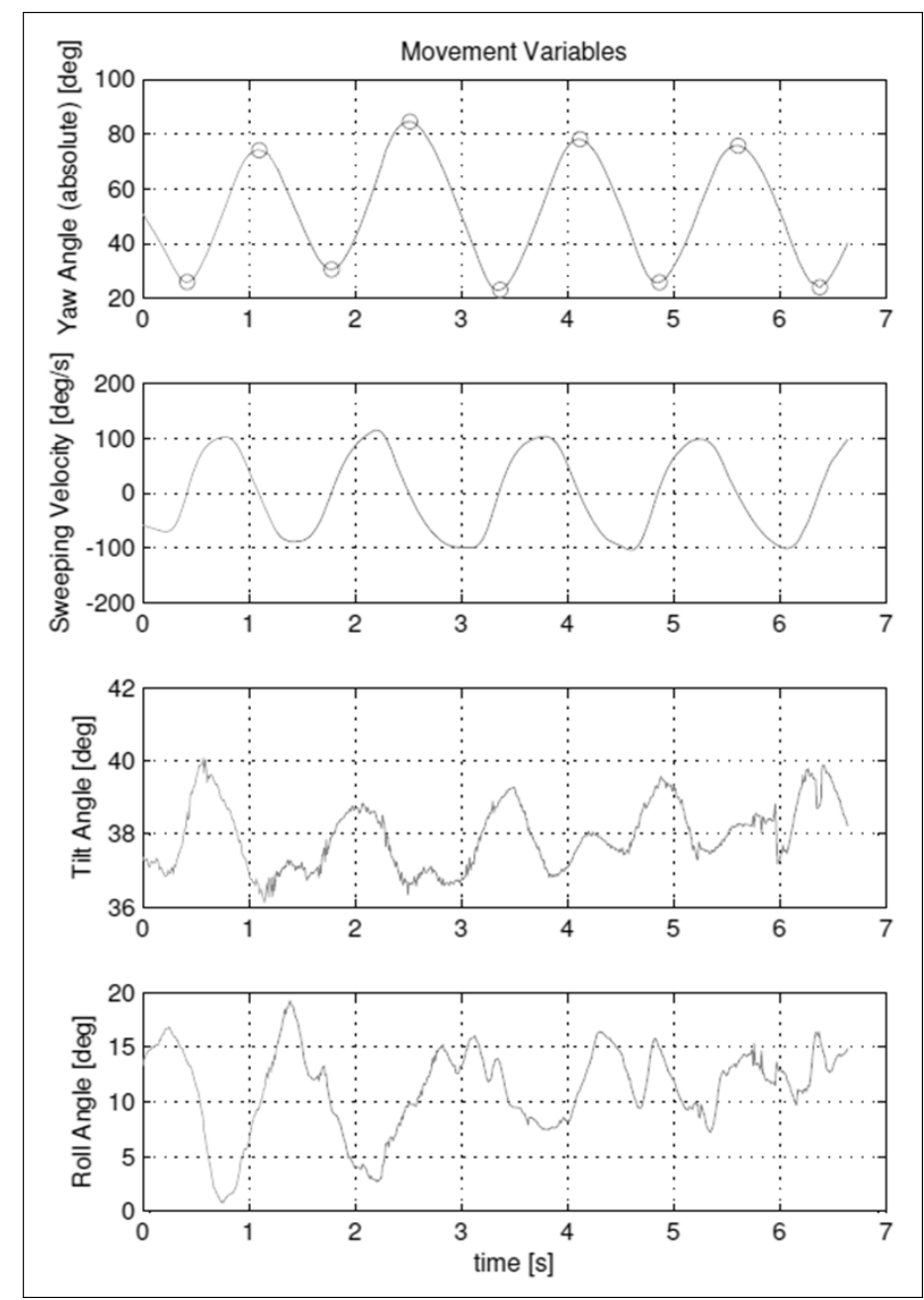

Fig. 4. Synchronized angles recorded from a representative subject while walking with the instrumented long cane with optical makers.

\section{Discussion and conclusion}

In this paper, usage variables of the constant contact technique focused on cane rotation around the cane axis, sweeping and grasping characteristics with the constant contact technique were investigated. The purpose of our experiments was not only to provide a detailed understanding of long cane usage of visually impaired persons, but also to derive guidelines for the development of efficient ETAs to complement the conventional long cane, which is still the most widely used navigation tool for blind and visually impaired persons.

The distance between the heel and the cane tip provides a range in which low obstacles can be detected with the long cane. This was found to be limited to below $1.45 \mathrm{~m}$ in all participants. With current sensor technology, an ETA can extend obstacle detection ranges and provide obstacle information over different 
vertical ranges such as at the head level, trunk level, lower extremity level, or detect drop-offs at a distance (Pyun et al., 2013).

In order to optimally select and design sensing elements for ETAs, the orientation angles of the long cane can be a crucial factor, especially when the ETA is integrated into the long cane. The mean tilt angle helps determine the ideal position and orientation of sensors of the ETA to be attached to the cane. As the experimental results showed relatively small variation of tilt angle, a fixed sensor on the long cane is able to detect obstacles reliably while employing the constant contact cane technique. Analogous to the tilt angle, the cane roll angle should also be considered when designing sensor elements in an ETA. If this angle shows high variability, the chance that a sensor detects the ground as obstacle or provides wrong information on the height of an obstacle increases. To alleviate this problem, sensors such as a gyroscopes, accelerometers, or tilt sensors can provide additional information about the rotation of the cane. However, the small range $\left(26.34^{\circ}\right)$ of measured mean roll angle may prevent false detection of the ground as a close obstacle. From the sweeping angle, we can infer the minimal field of view required for the sensors of an ETA to allow users to walk confidently. In combination with an IMU, it is possible to detect obstacles on one's walking path and avoid alarming the user about dangerless obstacles that are to either side. In agreement with the experiment by Nakamura (1997), the walking speed found in our experiments using a conventional long cane is below that of sighted subjects $(1.5 \mathrm{~m} / \mathrm{s})$. The mean walking speed of the nine congenitally blind subjects in our study was found to lie between the congenitally blind group $(0.86 \mathrm{~m} / \mathrm{s})$ and late blind group $(1.11 \mathrm{~m} / \mathrm{s})$. This slight difference may be explained by the navigation skills of subjects, residual vision such as light and color perception, or the small number of subjects. Along with the walking speed, the sweeping frequency can provide a reference for the update rate of the obstacle information and display latency and duration of auditory or haptic stimuli provided by ETAs. There was a large variability among subjects. In other words, there exist interpersonal differences of long cane usage in terms of sweeping characteristics, which need to be addressed by allowing adjustment by the individual user of an ETA. The sweeping velocity can also be useful to automatically estimate the user's intention during navigation. In our previous research (Gallo et al., 2010), a threshold on the sweeping velocity was employed to automatically switch between two ETA modes such as a sweeping mode - simple obstacle distance information provided during walking - and a scanning mode - standing still to explore the environment, in which more detailed information was presented.

Several grasp types such as the index finger grasp, thumb grasp, pencil grasp, fingertip grasp, and whole hand grasp have been reported in literature depending on the employed cane technique and situation (Jacobson, 1993). Some orientation and mobility guidelines suggest that the index finger should be extended for improved use (Yamauchi et al., 2003; McConville et al., 1978). When the constant contact or two-point touch technique is adopted, the middle finger, ring finger and little finger are mostly 
wrapped around the cane to achieve a firm grasp. Similar to the differences between measured and recommended parameters for the two-touch cane technique (Bongers et al, 2002), our results of the grasping analysis also showed deviation from the recommended grasp taught during mobility skill training. Fifty six percent of participants placed the thumb and index finger on the side of the cane and the other subjects placed these two fingers differently. However, they maintained the same grasp during walking with the long cane $(84.5 \%$ for thumb and $67.8 \%$ for index finger). Also, more than $90 \%$ of the time the tips of both the thumb and index finger were in contact with the cane handle while walking through an obstacle-free environment. The positions of the fingers on the handle can denote crucial information for the design of a haptic display in order to optimize the haptic perception when the ETA is mounted on the handle of the cane and held by the user.

Two of the subjects who participated in this study primarily use the two-point touch technique. While this might have affected their performance with the constant contact technique, we are unable to explore this effect statistically due to the small sample size. Nevertheless, both participants stated that they are also familiar with the constant contact technique, and employ it occasionally. We therefore assumed that there was no difference in their performance compared to the other participants. However, this would need to be confirmed in a dedicated study.

Based on the results of our experiments, guidelines to develop effective ETAs can be derived by considering the following long cane usage characteristics:

- The cane tilt angle is independent of the subject's height and the tilt range shows small variability. Therefore, the position of a sensor on the long cane to detect obstacles can be determined based purely on the sensor characteristics.

- $\quad$ There are large differences in mean sweeping angle and frequency, but intra-subject variability is low. Additional sensors (e.g. inertial sensors) might be required to trigger obstacle detection in the walking direction, and to offer the possibility of adapting the values to the individual user.

- Both intra and inter-subject variability of the roll angle range of the cane are rather small, but the initial handle orientation is important to assure that any sensor attached to the cane faces in the right direction. The design of the cane should allow adjustment of the cane rotation, or "enforce" a specific holding orientation through an adapted shape of the handle.

- $\quad$ The grasp type shows large inter-subject variability. However, fingers are mostly kept in contact with the cane handle during navigation with the long cane and intra-subject variability is low. Therefore, a wearable haptic device such as a thimble or ring type vibrotactile display that can be fixed on the cane handle could cope with the variability in grasp types, allowing individual adjustment by the user during the first use. 
In persons with visual loss, the long cane plays a crucial role in establishing a link between the user and the environment. The user can detect obstacles via haptic (physical contact) and auditory (semi-active echolocation) channels during a conventional long cane usage. In the case of an ETA, this link is replaced with a sensor, which measures the distance to an obstacle and presents obstacle information to the user via an auditory or haptic interface. Therefore, parameters of long cane usage such as the cane orientation, which informs on the optimal sensor location, and the sweeping frequency, which informs on the necessary update rate of the sensors are crucial parameters for the design of efficient ETAs. Moreover, knowledge about finger positions and cane contact during navigation will inform the design of displays for haptic feedback to deliver rich information about the environment from the cane (respectively the ETA) to the user. Studies with dedicated hardware will need to be carried out to validate the design principles proposed in this paper in the context of an ambulation task with obstacles. This will be the focus of our future work.

The results of our study showed a high inter-subject variability for many of the parameters, but with low intra-subject variability. Therefore, the ideal ETA will consider parameters of white cane usage and allow for a user-specific adaptation. We consider these results to serve as basis for a more user-centered approach to the design of future ETAs.

\section{ACKNOWLEDGEMENTS}

This research was supported by the Swiss Commission for Technology and Innovation, Project 11393.1 PFNM-NM and the National Center of Competence in Neural Plasticity and Repair of the Swiss National Science Foundation. The authors would like to thank all the participants who took part in the experiments, as well as Daniele Corciulo for his valuable comments during the preparation of the experiments. We also thank the Sensorimotor Systems Lab at ETH Zurich for allowing us to use their optical tracking system and providing support with the measurements. 


\section{REFERENCES}

Ambutech, Available from: http://www.ambutech.com/iglasses [Accessed 20 January 2016].

Benjamin, J Jr., 1971. The laser cane. Bionic instruments . Inc.,221 Rock Hill Road Bala Cynwyd, Pennsylvania 19004. pp. 443-450.

Bissitt, D., and Heyes, A. D., 1980. An application of bio-feedback in the rehabilitation of the blind. Applied Ergonomics 11.1, 31-33.

Black, T., 2009. Derivations of applied mathematics. http://www.derivations.org/derivations.pdf

Bongers, R. M., Schellingerhout, R., van Grinsven, R., \& and Smithsman, A. W., 2002. Variables in the touch technique that influence the safety of cane walkers. Journal of Visual Impairment and Blindness, 96(7), 516-531.

BrailleNote GPS, The Sendero Group LLC. Available from: http://www.senderogroup.com. [Accessed 20 January 2016].

Chandler, E., Worsfold, J., 2013. Understanding the requirements of geographical data for blind and partially sighted people to make journeys more independently. Applied ergonomics, 44(6), 919928.

Clark-Carter, D., Heyes, A., Howarth, C., 1986. The efficiency and walking speed of visually impaired people. Ergonomics, 29, 779-789.

Cutter J., 2007. TRAVEL/O\&M - Functions of the cane and the bottom-up approach to O\&M for children. Future Reflections. The National Federation of the Blind Magazine for Parents and Teachers of Blind Children, 26.

De Bruin, D., 1981. The constant contact technique. American Association of Workers for the Blind Newsletter, 9, 23-31.

Farcy, R., Bellik, Y., 2002. Locomotion assistance for the blind. Universal Access and Assistive Technology, Springer, 277-284.

Foundation Access for All. http://www.access-for-all.ch/ [Accessed 20 January 2016].

Gallo, S., Chapuis, D., Santos-Carreras, L., Kim, Y., Retornaz, P., Bleuler, H., Gassert, R., 2010. Augmented long cane with multimodal haptic feedback. IEEE/RAS-EMBS International Conference on Biomedical Robotics and Biomechatronics, 149-155.

GDP Research Australia. MiniGuide. Available from: http://www.gdp-research.com.au. [Accessed 20 January 2016].

Goldstein, B., Wiener, W., 1981. Acoustic analysis of the Sonic Guide. Journal of the Acoustical Society of America, 70, 313-320.

Heyes, A. D., 1984. Sonic Pathfinder: A programmable guidance aid for the blind. Wireless World, 90, 26-29. 
Hill, E., Ponder, P., 1976. Orientation and mobility techniques: A guide for the practitioner. New York (NY): American Foundation for the Blind.

Hoyle, B., Waters, D., 2008. Mobility AT: The Batcane (UltraCane). Assistive technology for visually impaired and blind people: Springer, 209-229.

Jacobson, W. H., 1993. The art and science of teaching orientation and mobility to persons with visual impairments. American Foundation for the Blind (AFB) Press.

Johnson, J. T., Johnson, B. F., Blasch, B. B, de l'Aune, W. D., 1998. Gait and long cane kinematics: a comparison of sighted and visually impaired subjects. Journal of Orthopaedic \& Sports Physical Therapy, 27, 162-166.

Kay, L., 1964. An ultrasonic sensing probe as a mobility aid for the blind. Ultrasonics, 2, 53-59.

Kim, D., Emerson, R.W, S., Curtis, A. B., 2009a. Drop-off detection with the long cane: effects of different cane techniques on performance. Journal of Visually Impaired and Blind, 103, 519-530.

Kim, L., Park, S., Lee, S., Ha, S., 2009b. An electronic aid for a visually impaired person using an ultrasonic sensor. IEICE Electronics Express, 6, 794-799.

Kim, Y., Harders, M., Gassert, R., 2015. Identification of Vibrotactile Patterns Encoding Obstacle Distance Information. IEEE Transactions on Haptics, 8(3), 298-305.

Koestler, F. A., 2004. The unseen minority: A social history of blindness in the United States. AFB Press.

K-Sonar. Available from: http://www.ksonar.com/history.php [Accessed 20 January 2016].

Kulyukin, V., Gharpure, C., Sute, P., De Graw, N., Nicholson, J., 2004. A robotic wayfinding system for the visually impaired. Proceedings of the 16th Conference on Innovative Applications of Artifical Intelligence, 864-869.

LaGrow, S., 1999. The use of the sonic pathfinder as a secondary mobility aid for travel in business environments: a single-subject design. Journal of Rehabilitation Research and Development, 36, 333-340.

LaGrow, S., Weessies, M., 1994. Orientation and mobility: Techniques for Independence. Palmerston North, New Zealand: Dunmore Press.

Lee, C. L., Chen, C. Y., Sung, P. C., Lu, S. Y., 2014. Assessment of a simple obstacle detection device for the visually impaired. Applied ergonomics, 45(4), 817-824.

Leuenberger, K., Gassert, R., 2011. Low-Power Sensor Module for Long-Term Activity Monitoring. IEEE Engineering in Medicine and Biology Society, 2237-2241.

McConville, B., Fowler, P., Schur, S., 1978. Orientation \& Mobility. Texas state commission for the blind assessment-placement-training system training guides for the blind-multihandicapped, ARBEC Inc. 
Meers, S., Ward K., 2005. A vision system for providing the blind with 3d colour perception of the environment.

Nakamura, T., 1997. Quantitative analysis of gait in the visually impaired. Disability and Rehabilitation, 19, 194-197.

Pyun, P., Kim, Y., Wespe, P., Schneller, S., Gassert R., 2013. Advanced Augmented White Cane with Obstacle Height and Distance Feedback. IEEE International Conference on Rehabilitation Robotics, 1-6. doi: 10.1109/ICORR.2013.6650358.

Ramsey, V., Blasch, B., Kita, A., Johnson, B., 1999. A biomechanical evaluation of visually impaired persons' gait and long-cane mechanics. Journal of Rehabilitation Research and Development, 36, $323-332$.

Sánchez, J., Aguayo, F., Hassler, T., 2007. Independent outdoor mobility for the blind. International Conference on Virtual Rehabilitation, 114-120.

Schenkman, B. N., Jansson, G., 1986. The detection and localization of objects by the blind with the aid of Long-cane Tapping Sounds. Human Factors, 28, 607-618.

Shoval, S., Borenstein, J., Koren, Y., 1998. The Navbelt - A Computerized travel aid for the blind based on mobile robotics technology. IEEE Transaction on Biomendical Engineering, 45, 1376-1386.

Ulrich, I., Borenstein, J., 2001. The GuideCane - Applying mobile robot technologies to assist the visually impaired. IEEE Transactions on Systems, Man, and Cybernetics, - Part A: Systems and Humans, 31, 131-136.

Wall, R. S. and Ashmead D.H., 2002. Biomechanical movements in experienced cane users with and without visual impairments. Journal of visual impairment \& blindness, 501-515.

Wang, Y., Kuchenbecker, KJ., 2012. HALO: Haptic Alerts for Low-hanging Obstacles in white cane navigation. IEEE Haptics Symposium, 527-532.

WHO. 2010. Action plan for the prevention of avoidable blindness and visual impairment 2009-2013. http://www.who.int/blindness/ACTION_PLAN_WHA62-1-English.pdf [Accessed 20 January 2016].

Yamauchi, S., Yamauchi, Y., Senta, K., Rengo, N. , 2003. Tactile ground surface indicators for blind persons. National Rehabilitation Center for Persons with Disabilities. Japan Federation of the Blind. 